\title{
Co-localization of the Putative Histone Acetylase Spt10p and Chemically Modified Histone H3
}

\author{
Tiffany A. Pompa ${ }^{1}$, Jonathan Blaize ${ }^{1}$, William L'Amoreaux ${ }^{1,2}$ and Chang-Hui Shen ${ }^{1,2}$ \\ ${ }^{1}$ Department of Biology, College of Staten Island, 2800 Victory Blvd. Staten Island, New \\ York 10314 \\ ${ }^{2}$ City University of New York, Institute for Macromolecular Assemblies, College of \\ Staten Island, 2800 Victory Blvd. Staten Island, New York 10314
}

Transcription is one of the most important cellular activities in eukaryotic cells. Two chromatin remodeling activities may involve gene activation through chromatin structure. One is the chromatin remodeling complex which can reconfigure the nucleosome positioning and the other is the histone acetylation which can reduce the interaction between the histone and DNA. Both activities can help transcription factors gain access to their binding sites and thus activate gene transcription. We have discovered that Spt10p is a putative histone acetylase, since the targeted histone acetylation is only observed in the presence of Spt10p. To further understand the significance of Spt10p in gene expression and its connection with cell cycle activity, immunofluorescence technique was employed in this study. In these studies, the cells were synchronized to late $\mathrm{G} 1$ of the cell cycle using $10 \mathrm{mg} / \mathrm{ml}$ hydroxyurea. The cells were spheroplasted using $0.1 \mathrm{mg} / \mathrm{ml}$ lyticase and prepared for immunohistochemistry. The primary antibodies were directed against a) an HA-tagged Spt10p and b) either an acetylated histone $\mathrm{H} 3$ or a phosphorylated histone H3. The Spt10p was indirectly localized using an anti-mouse IgG conjugated with Texas Red and the $\mathrm{H} 3$ was indirectly localized using an anti-rabbit IgG conjugated with FITC. All cells were imaged using a Leica SP2 AOBS confocal microscope. Confocal microscopy revealed that in the wild type Spt10p is co-localized with phosphorylated or acetylated histone H3. Our data showed that they were co-localized around the rim of the nucleus suggesting that to localize the proteins in the nucleus will require synchronization of the cell cycle. These results suggest that Spt 10p may play an important role in histone modification during gene activation. In the Spt10 deletion mutation cells (Spt10 ), only the histone H3 was localized in the cells, confirming the success of the deletion mutation. 


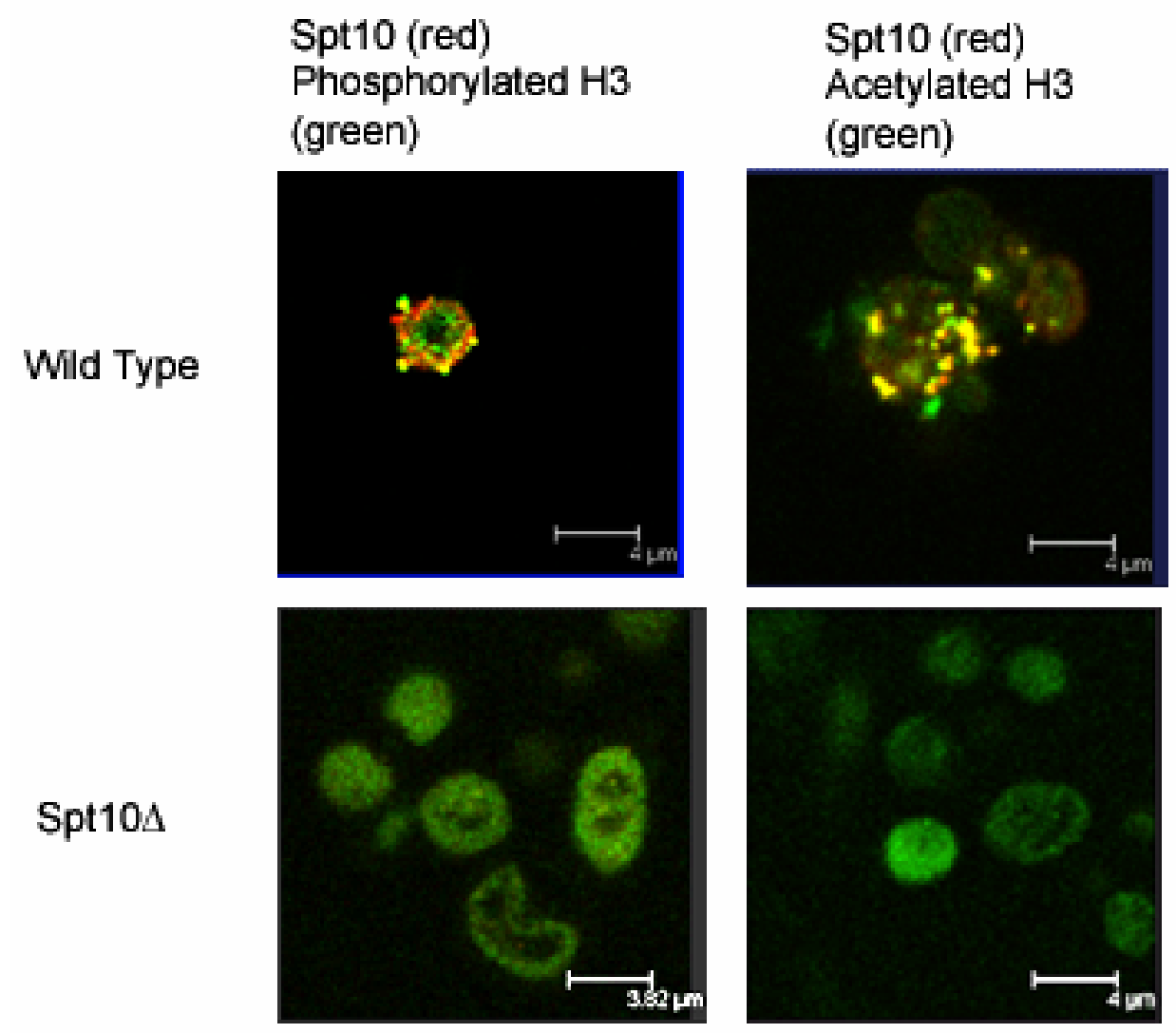

